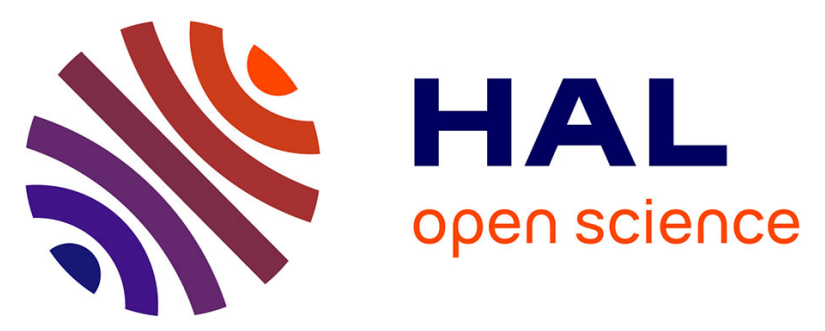

\title{
Sources de variation et reproductibilité de la mesure des érosions de la polyarthrite rhumatoïde par HRpQCT
}

\author{
Nada Ibrahim-Nasser, Hubert Marotte, Antoine Valery, Carine Salliot,
} Hechmi Toumi, Éric Lespessailles

\section{- To cite this version:}

Nada Ibrahim-Nasser, Hubert Marotte, Antoine Valery, Carine Salliot, Hechmi Toumi, et al.. Sources de variation et reproductibilité de la mesure des érosions de la polyarthrite rhumatoïde par HRpQCT. Revue du Rhumatisme, 2019, 86, pp.170 - 177. 10.1016/j.rhum.2018.09.006 . hal-03486213

\section{HAL Id: hal-03486213 \\ https://hal.science/hal-03486213}

Submitted on 20 Dec 2021

HAL is a multi-disciplinary open access archive for the deposit and dissemination of scientific research documents, whether they are published or not. The documents may come from teaching and research institutions in France or abroad, or from public or private research centers.
L'archive ouverte pluridisciplinaire HAL, est destinée au dépôt et à la diffusion de documents scientifiques de niveau recherche, publiés ou non, émanant des établissements d'enseignement et de recherche français ou étrangers, des laboratoires publics ou privés.

\section{(ㅇ)(1) $\$$}

Distributed under a Creative Commons Attribution - NonCommerciall 4.0 International 


\section{Sources de variation et reproductibilité de la mesure des érosions de la polyarthrite rhumatoïde par HRPQCT}

Nada Ibrahim-Nasser ${ }^{1}$, Hubert Marotte ${ }^{2,3}$, Antoine Valery ${ }^{4}$, Carine Salliot ${ }^{1}$, Hechmi Toumi $^{5}$, Éric Lespessailles ${ }^{1,5}$

1 Service de rhumatologie, centre hospitalier régional d'Orléans, 1 rue Porte Madeleine, 45032 Orléans, France

${ }^{2}$ INSERM U1059, SAINBIOSE, université de Lyon, 42023, Saint-Étienne, France

${ }^{3}$ Département de rhumatologie, université de Saint-Étienne, 42055 Saint-Étienne, France

${ }^{4}$ Service du D.I.M, centre hospitalier régional d'Orléans, 1 rue Porte Madeleine, 45032 Orléans, France

${ }^{5}$ I3MTO, EA 4708, université d'Orléans, 45032 Orléans, France

\section{Auteur correspondant :}

Nada Ibrahim-Nasser

E-mail : nadaii@hotmail.fr

Tél. : 0033238514858 


\section{$\underline{\text { Résumé }}$}

Objectifs. Évaluer la reproductibilité et les sources de variation dues au repositionnement pour la mesure manuelle des érosions des articulations métacarpophalangiennes (MCP) par scanner périphérique à haute résolution (HRpQCT) dans la polyarthrite rhumatoïde (PR).

Méthodes. Des patients atteints de PR et présentant au moins une érosion au niveau de la deuxième, troisième ou quatrième MCP à la radiographie conventionnelle ont été inclus. Deux scanners ont été réalisés le même jour, avec un repositionnement. Le critère d'évaluation principal était le calcul de la précision à court terme du diamètre, de la profondeur et du volume des érosions. Les critères secondaires étaient la précision intra- et interopérateur, la plus petite variation significative et les sources de variation des mesures.

Résultats. Vingt-neuf patients ont été inclus et 406 érosions de 0,9 à $3 \mathrm{~mm}$ de diamètre ont été analysées. Les coefficients de corrélation intraclasse (ICC) pour la précision de la mesure du diamètre axial, de la profondeur axiale et du volume après repositionnement étaient de 0,8,0,96 et 0,99, respectivement. Les valeurs de RMS CV et RMS SD étaient respectivement de $16 \%, 0,26 \mathrm{~mm} ; 17,5 \%, 0,32 \mathrm{~mm}$ et $19,7 \%, 0,93 \mathrm{~mm}^{3}$. Les ICC de la précision intra-opérateur étaient de 0,92, 0,97 et 0,99 avec une RMS CV respective de $16 \%, 16,4 \%$ et de $18,7 \%$. En ce qui concerne le volume, la précision interopérateur était de 0,99 avec une RMS CV de $14 \%$. La plus petite variation significative pour le diamètre, la profondeur et le volume était de $0,3 \mathrm{~mm}, 0,2 \mathrm{~mm}$ et $0,3 \mathrm{~mm}^{3}$. Aucune corrélation significative avec les paramètres de micro-architecture osseuse n'a été établie.

Conclusion. L'analyse par HRpQCT est une méthode reproductible pour caractériser et mesurer les érosions, sans que le repositionnement n'ait d'incidence. Nous avons toutefois observé une faible précision de la mesure manuelle, imputable à la variabilité intra-opérateur.

Mots-clés : polyarthrite rhumatoïde, érosions, HRpQCT, précision 


\section{Introduction}

La polyarthrite rhumatoïde (PR) est une maladie articulaire auto-immune chronique qui touche environ $1 \%$ de la population mondiale et se définit par la présence de synovite et d'érosions osseuses [1]. En radiographie conventionnelle, une érosion osseuse se présente sous la forme d'une rupture corticale résultant de l'action de cytokines sur la différenciation des ostéoclastes, privilégiant les zones d'insertions ligamentaires [2]. Ces lésions, qui apparaissent très tôt dans la maladie, ne régressent jamais spontanément [3,4]. Elles sont liées à l'activité de la maladie et leur évolution constitue un facteur de pronostic défavorable [5]. II est donc souhaitable de les détecter le plus tôt possible, avec une grande précision permettant de quantifier les petits changements. Par ailleurs, des traitements très efficaces de la PR se développent, tels que les inhibiteurs du facteur de nécrose tumorale anti-TNF, ce qui impose de pouvoir surveiller les effets liés à ces traitements [6,7].

Dans les études cliniques, l'examen de référence pour évaluer les érosions osseuses est la radiographie conventionnelle [8,9]. Or, bien que hautement spécifique, cette méthode n'a qu'une faible sensibilité car les rayons sont projetés en deux dimensions [8,9]. Si l'échographie, technique sans irradiation, est décrite comme plus sensible que la radiographie [10], elle ne permet pas d'atteindre toutes les surfaces osseuses et sa spécificité a été décrite comme plus faible que celle du scanner périphérique, avec $28 \%$ de faux positifs [10,11]. D'autres études ont montré une meilleure sensibilité de l'IRM qui a permis de quantifier le volume des érosions manuellement ou par des techniques automatisées [12-15]. Cependant, l'IRM est une technique difficile d'accès et la présence d'œdème osseux ou d'artefacts peut conduire à sous-estimer les érosions [12,15].

Le scanner périphérique à haute résolution (HRpQCT) a été mis au point initialement afin de quantifier la micro-architecture osseuse trabéculaire au tibia distal et au radius in vivo [16]. Des études ont récemment examiné les dégradations microarchitecturales et les érosions osseuses des articulations métacarpophalangiennes (MCP) de patients atteints de PR $[17,18]$. La sensibilité élevée de cette technique a permis d'évaluer des ruptures corticales de moins de $500 \mu \mathrm{m}$ [19]. En outre, les érosions identifiées par HRpQCT étaient beaucoup plus nombreuses qu'avec la 
radiographie conventionnelle [20]. Cependant, l'inflammation synoviale n'a pas été évaluée par la technique HRpQCT, actuellement utilisée dans des projets de recherche car seuls quelques centres en disposent. La mesure quantitative de la largeur, de la profondeur et du volume des érosions par HRpQCT a été décrite $[6,21,22]$.

Toutefois, une évaluation de grande ampleur de la reproductibilité et des sources de variation est nécessaire. L'objectif de notre étude était d'évaluer la précision à court terme après repositionnement et les sources de variation de la mesure quantitative manuelle des érosions par HRPQCT.

\section{Méthodes}

\subsection{Patients}

Les patients de cette étude ont été recrutés dans le service de rhumatologie du centre hospitalier d'Orléans et remplissaient les critères 2010 de l'ACR/EULAR pour la PR. Pour être inclus, ils devaient présenter au moins une érosion au niveau de la deuxième, troisième ou quatrième MCP sur des radiographies conventionnelles. Les femmes enceintes, les personnes mineures et celles qui se trouvaient dans l'incapacité de donner leur consentement éclairé ont été exclues. Deux examens d'imagerie ont été réalisés le même jour pour chaque patient, avec repositionnement complet entre les deux. L'étude a été réalisée conformément à la Déclaration d'Helsinki. L'approbation du comité d'éthique local de Tours (CPP 2013-R2, IDRCB 2012-A00026-39) a été obtenue.

Nous avons collecté des données cliniques et biologiques : durée des symptômes, score d'activité de la maladie (DAS 28), présence ou absence de facteur rhumatoïde (FR) et d'anticorps antipeptides cycliques citrullinés (ACPA) au moment du diagnostic, ainsi que les dernières valeurs de vitesse de sédimentation (VS) et de protéine réactive $\mathrm{C}(\mathrm{CRP})$.

Procédure d'imagerie 
Un scanner HRpQCT (XtremeCT, Scanco Medical AG, Bassersdorf, Suisse) a été réalisé sur les articulations MCP (2-4) de la main présentant le plus d'érosions. Cette technique permet l'acquisition simultanée de 110 coupes parallèles avec une taille des voxels isotropiques de $82 \mu \mathrm{m}$. II a déjà été montré que les érosions touchent souvent la tête métacarpienne [10,19,23]. Par ailleurs, les érosions de la cinquième MCP ont été décrites comme plus spécifiques de la PR à l'échographie mais n'avaient pas encore été étudiées par HRpQCT [11]. Pour ces raisons et pour que la région d'intérêt apparaisse sur le même topogramme, 248 coupes au total ont été acquises, à partir d'une ligne de référence située à l'extrémité de la troisième tête métacarpiennne et en poursuivant en direction proximale sur 20,3 mm. La région d'intérêt couvrait la totalité des têtes du deuxième au quatrième métacarpien, la partie distale de la cinquième tête (168 coupes) et la partie proximale des quatrième et cinquième bases phalangiennes (80 coupes). La dose efficace était d'environ $3 \mu \mathrm{Sv}$ par mesure et la durée de balayage de 8 minutes.

Pour chaque patient, nous avons mesuré l'épaisseur corticale (C.Th, en $\mu \mathrm{m}$ ), la fraction volumique de l'os trabéculaire (BV/TV, en \%), le nombre de travées (Tb.N, en $\mathrm{mm}^{-1}$ ), leur épaisseur (Tb.Th, en $\mu \mathrm{m}$ ) et la séparation entre les travées (Tb.Sp, en $\mu \mathrm{m}$ ) au niveau de la deuxième tête métacarpienne [16-18].

\subsection{Score d'érosion}

Les érosions ont été définies comme des lésions osseuses marginales à bords nets, localisées en juxta-articulaire avec une rupture corticale visible sur au moins deux coupes adjacentes et dans deux plans perpendiculaires [24]. Les érosions typiques de la $P R$ forment un « $U$ » évasé [25]. Toutes les ruptures corticales ne sont pas des érosions, et on observe fréquemment des lésions tubulaires qui sont en réalité des vaisseaux $[12,26]$. L'aspect des ostéophytes en coupe axiale peut aussi évoquer une érosion [25]. Si l'on peut observer des érosions de moins de $0,5 \mathrm{~mm}$ chez des sujets en bonne santé, au-delà de $1,9 \mathrm{~mm}$ en revanche, elles sont hautement spécifiques de la PR [19]. Par une méthode semi-automatisée, Emond a exclu les érosions couvrant plus de la moitié de la tête métacarpienne car elles ne sont pas présentes 
chez les PR débutantes et sont irréversibles [15]. C'est pourquoi nous avons conservé les érosions de 0,9 à $3 \mathrm{~mm}$ de diamètre pour notre étude, en nous concentrant sur celles de plus de $1,9 \mathrm{~mm}$.

Nous avons utilisé le logiciel d'imagerie médicale DICOM (Digital Imaging and Communication in Medicine) OsiriX 6.0 pour évaluer l'emplacement et les mesures quantitatives de chaque érosion en suivant la description de certains auteurs $[6,21,27]$. La distribution de fréquence des lésions a été analysée dans quatre quadrants : palmaire, ulnaire, dorsal et radial. Le diamètre et la profondeur de chaque érosion ont été mesurés dans les plans axial (figure $1 A$ ) et perpendiculaire (figure 1B). Pour évaluer le volume, nous avons délimité l'érosion manuellement coupe par coupe dans le plan axial en utilisant le volume de la région d'intérêt enregistré dans le logiciel (figure 1C) [4]. En IRM, la délimitation manuelle a été décrite comme la technique de référence pour la mesure du volume $[14,15]$.

\subsection{Analyse statistique}

Les analyses statistiques ont été effectuées à l'aide des logiciels $R$ version 3.1.2 et Rstudio version 0.98.1091. Les valeurs de $p<0,05$ ont été considérées comme significatives. Nous avons exprimé la reproductibilité à l'aide du coefficient de corrélation intraclasse (ICC) [28]. De plus, pour chaque patient, un coefficient de variation (CV) a été calculé à partir de l'écart-type (SD) de deux mesures répétées, divisé par la moyenne pour ce patient. L'écart-type a été défini par la formule :

$$
S D=\sqrt{\frac{\left[(\mathrm{x} 1-\mathrm{n})^{2}+(\mathrm{x} 2-\mathrm{n})^{2}\right]}{N-1}}
$$

où « $N$ » correspondait au nombre de mesures, « $x 1$ » et « $x 2$ » aux valeurs du paramètre évalué avant et après repositionnement et « $\mathrm{n}$ » à la moyenne des deux mesures. La racine carrée des coefficients de variation, RMS CV, et la racine carrée de l'écart-type, RMS SD, ont ensuite été calculées, comme décrit par Glüer et al. [29]. Les petits changements peuvent donner lieu à des coefficients de variation élevés, tandis que l'écart-type donne l'écart absolu, qui constitue la meilleure approche statistique selon Grampp [30]. La méthode de Bland et Altman a été appliquée à l'évaluation qualitative du biais de l'erreur de précision dans les mesures 
de largeur, de profondeur et de volume des érosions, en calculant la différence moyenne entre les deux mesures (le biais) et les limites d'agrément à $95 \%$ comme la différence moyenne $\pm 1,96$ SD des différences. Ces limites devraient inclure $95 \%$ des différences entre les deux mesures [31,32].

Pour l'évaluation de la reproductibilité à court terme, les deux scanners de chaque patient ont été lus en aveugle par un lecteur (NIN). L'évaluation de la fiabilité intraopérateur a été réalisée en mesurant 30 érosions sélectionnées de manière aléatoire sur les différents clichés, trois mois plus tard. Pour la reproductibilité interobservateur, les premiers clichés ont été lus par un autre rhumatologue (HM). La plus petite variation significative (PPVS) détectable a été calculée sur la base de 35 érosions individuelles par la formule suivante [33] : PPVS $=1,96 \times \sqrt{2} \times \mathrm{SD}$. Nous avons étudié la corrélation entre la variation de la mesure (exprimée en RMS SD) et les données cliniques à l'aide du coefficient de corrélation de Pearson. Le lien entre la taille et l'emplacement des érosions a également été étudié, de même que la corrélation avec les paramètres microstructurels.

\subsection{Rôle de la Source de financement}

Non applicable. Les auteurs déclarent n'avoir bénéficié d'aucun financement ni soutien commercial.

\section{Résultats}

Vingt-neuf patients (24 femmes) ont été inclus dans l'étude. L'âge moyen $\pm E T$ était de 56,7 $\pm 12,4$ ans et la durée de la maladie de 14,1 $\pm 9,57$ ans. Le score DAS 28 était de $2,71 \pm 1,46$. La présence de FR et d'ACPA a été retrouvée respectivement chez $77 \%$ et $67 \%$ des patients. Des glucocorticoïdes, des csDMARD et des bDMARD étaient administrés respectivement à $31 \%, 72 \%$ et $76 \%$ des patients. Au total, 406 érosions ont été identifiées, $14 \pm 11,1$ par patient. Le diamètre axial, la 
profondeur axiale et le volume étaient respectivement de 1,55 0,53 mm, $1,88 \pm 1,73 \mathrm{~mm}$ et $5,2 \pm 13 \mathrm{~mm}^{3}$. Cent une érosions mesuraient plus de 1,9 $\mathrm{mm}$ de large. Les têtes de la MCP radiale étaient les plus touchées, avec une prédominance dans les deuxième et troisième têtes de MCP (figure 2).

Les résultats de reproductibilité à court terme après repositionnement sont présentés dans le tableau 1. L'ICC s'échelonnait de 0,8 à 0,99 et la RMS CV de 16 à 23,5\%. Pour les érosions de diamètre supérieur à $1,9 \mathrm{~mm} \quad(n=101)$, la RMS CV et la RMS SD de la largeur axiale, de la profondeur et du volume étaient de 16,6\%, $0,34 \mathrm{~mm} ; 13,5 \%, 0,41 \mathrm{~mm}$ et $14 \%, 1,45 \mathrm{~mm}^{3}$, respectivement, à comparer avec $16,6 \%, 0,22 \mathrm{~mm} ; 18,6 \%, 0,29 \mathrm{~mm}$ et $21,2 \%, 0,67 \mathrm{~mm}^{3}$ pour les petites érosions $(n=305)$.

Les graphiques de Bland et Altman pour chaque paramètre sont présentés à la figure 3. Les limites d'agrément étaient relativement étroites, avec une différence moyenne proche de 0 suggérant un agrément élevé des deux mesures [32]. Quelle que soit la taille de l'érosion, les résultats étaient linéaires, sans erreur systématique. Tous les graphiques avaient la même forme, avec une majorité de points sur la gauche car la plupart des érosions de l'échantillon étaient de petite taille. Les résultats de reproductibilité intra-opérateur pour le diamètre axial et la profondeur axiale, le diamètre et la profondeur perpendiculaires et pour le volume étaient de $16 \%, 0,29 \mathrm{~mm} ; 16,4 \%, 0,17 \mathrm{~mm} ; 19,2 \%, 0,43 \mathrm{~mm} ; 17 \%, 0,23 \mathrm{~mm}$ et $18,7 \%$, $1,24 \mathrm{~mm}^{3}$. Les ICC respectifs étaient de 0,92,0,97,0,93,0,94 et 0,99. En ce qui concerne la précision interopérateur du volume, I'ICC était de 0,99 avec une RMS CV de $14 \%$.

Les plus petites variations significatives (PPVS) pour le diamètre axial et la profondeur axiale, la largeur et la profondeur perpendiculaires et pour le volume étaient de $0,33 \mathrm{~mm}, 0,22 \mathrm{~mm}, 0,35 \mathrm{~mm}, 0,43 \mathrm{~mm}$ et $0,33 \mathrm{~mm}^{3}$ respectivement.

Pour évaluer les sources de variation des mesures, nous avons calculé la RMS SD par topographie et par patient, et recherché une corrélation avec les données cliniques (âge, durée de la maladie et score DAS 28), les caractéristiques des érosions (taille et topographie), les paramètres micro-architecturaux et l'expérience du lecteur. Les résultats sont présentés dans le tableau 2. Sur le plan des données 
de la PR, nous avons constaté que l'âge était significativement corrélé à la variabilité des mesures axiales $(r=0,44[0,09 ; 0,70] ; r=0,40[0,04 ; 0,67] ; p<0,05)$ et que la durée des symptômes était significativement corrélée à la variabilité des mesures de largeur perpendiculaire et de volume $(r=0,46[0,12 ; 0,71] ; r=0,39[0,02 ; 0,66]$; $p<0,05)$. En ce qui concerne les caractéristiques des érosions, la taille était significativement et positivement corrélée à la variabilité de toutes les mesures. Nous n'avons identifié aucune corrélation avec la localisation dans différents quadrants ou sur différentes articulations. Aucune corrélation significative n'a été constatée avec les paramètres de micro-architecture osseuse. Pour évaluer si l'entraînement pouvait améliorer la précision, nous avons comparé la RMS SD des premiers et des derniers patients (selon l'ordre d'inclusion) étudiés. Les RMS SD par patient étaient généralement proches, à l'exception de quelques patients mais indépendamment de l'ordre de lecture (figure 4).

\section{Discussion}

Nous rapportons ici les résultats de la première étude à évaluer l'effet du repositionnement dans la mesure des érosions par scanner HRpQCT chez des patients atteints de PR. La présente étude a démontré que la mesure des érosions par HRpQCT est hautement reproductible, avec un ICC de 0,8 à 0,99 pour l'évaluation de la reproductibilité à court terme avec repositionnement complet entre deux acquisitions. La reproductibilité intra-opérateur était bonne, avec un ICC de 0,92 à 0,99 et la reproductibilité interopérateur excellente $(0,99)$ pour la mesure du volume des érosions. Les RMS CV étaient moins satisfaisantes, allant jusqu'à $23,5 \%$ pour l'évaluation de la profondeur perpendiculaire des érosions. Nous n'avons trouvé aucune autre étude évaluant la précision à court terme avec repositionnement dans le contexte de l'évaluation des érosions dans la PR.

La reproductibilité doit être évaluée avant d'utiliser un dispositif dans une étude prospective. Ici, nous nous sommes concentrés sur le repositionnement car la position pourrait constituer une cause majeure d'imprécision. Glüer et al. [29] recommandent de répéter les mesures trois fois pour 14 patients ou deux fois pour 
27 patients [29]. Pour limiter l'exposition des patients aux rayons, nous avons procédé à deux acquisitions répétées pour 29 patients.

En ce qui concerne la reproductibilité intra-opérateur, nous avons obtenu un très bon ICC de 0,92 à 0,99 pour les différents paramètres de mesure des érosions, et des résultats très proches de ceux rapportés dans d'autres études [4,6,22]. En revanche, les RMS CV étaient plus élevées, s'échelonnant respectivement de $16 \%$ à $18,7 \%$ pour le diamètre axial et le volume, avec une RMS SD de 1,24 $\mathrm{mm}^{3}$ pour le volume. Srikhum et al. ont rapporté un ICC de 0,99 avec une RMS CV de 4,7\%, mais leur échantillon contenait 38 érosions de plus de $4 \mathrm{~mm}$ en largeur ou en profondeur [22]. Töpfer et al. ont obtenu une RMS CV de 11,58 \% avec une RMS SD de $1,16 \mathrm{~mm}^{3}$ pour la mesure manuelle des volumes avec reconstruction par demiellipses de 18 érosions [21]. Ils ont également rapporté une meilleure RMS CV pour les plus grandes érosions, mais avec une RMS SD plus élevée, comme dans notre étude [21].

Ces observations nous ont conduits à reconsidérer la pertinence de l'utilisation de la RMS CV dans la présente étude. Tout d'abord, la taille des érosions n'était pas distribuée normalement, ce qui peut avoir une incidence sur la RMS CV. Ensuite, les coefficients de variation sont directement influencés par la taille des érosions. Ainsi, pour un même coefficient de variation de $7 \%$, une érosion de $2,65 \mathrm{~mm}$ a été retrouvée à $2,41 \mathrm{~mm}$ après repositionnement, et une autre de $0,974 \mathrm{~mm}$, à $1,078 \mathrm{~mm}$. Par conséquent, étant donné que nous avions une majorité d'érosions inférieures à 1,9 mm de largeur, de très petites variations de la taille de l'érosion, non cliniquement pertinentes, ont pu être à l'origine de coefficients de variation élevés. La RMS SD semble donc plus pertinente que la RMS CV pour estimer la précision sans être influencée par la taille [29,30]. De plus, l'erreur de précision exprimée par le rapport de l'écart-type sur la valeur moyenne correspondante (coefficient de variation) n'est pas appropriée puisqu'elle ne prend pas en compte la plage physiologique des valeurs du paramètre mesuré ou les caractéristiques particulières d'une population distincte. II est donc préférable d'utiliser une technique de faible précision mais avec une plage de valeurs (sensibilité) importante plutôt qu'une technique plus précise mais moins sensible [34]. La sensibilité dépend de nombreux facteurs, y compris du type de technique et du site de mesure, aussi devons-nous 
standardiser nos résultats de précision par la méthode de l'ICC [28]. Les graphiques de Bland et Altman ont été corrélés aux ICC car ils ne faisaient apparaître aucune tendance ni erreur systématique. Les limites d'agrément étaient étroites et proches de 1, ce qui est cliniquement acceptable. Toutefois, le groupe SPECTRA (Study group for Xtreme CT in $R A$ ) a recommandé la publication des résultats de précision en RMS CV [35]. C'est pourquoi nous avons exprimé nos résultats en RMS CV, RMS SD et ICC.

Nous avons étudié au total 406 érosions de 0,9 à $3 \mathrm{~mm}$ de diamètre, un nombre considérablement plus élevé que les échantillons d'érosions rapportés par d'autres auteurs, qui comprenaient 18 à 72 érosions [6,7,21,22]. Le grand nombre d'érosions de la présente étude a été expliqué par différents facteurs. Tout d'abord, la durée d'évolution de la PR de la plupart de nos patients, plus de dix ans, a permis d'évaluer de nombreuses érosions. Ensuite, nous avons inclus les petites érosions (inférieures à $1,9 \mathrm{~mm}$ de diamètre dans $305 \mathrm{cas}$ ) car elles constituent la cible du traitement au début de la PR. Enfin, nous avons également inclus la cinquième MCP dans l'analyse $(n=61)$. Il a déjà été démontré que les érosions touchent de préférence le quadrant radial des deuxième et troisième têtes métacarpiennes $[2,19,20]$. Nous avons montré que la cinquième MCP était moins touchée mais obéissait à la même tendance de localisation des érosions dans le quadrant radial de la partie métacarpienne de l'articulation. Cela pourrait s'expliquer par les sites d'insertion des ligaments latéraux, indépendamment de la présence d'une synovite, comme décrit par Tan et al. [36]. Quel que soit l'emplacement des érosions, le scanner HRpQCT a permis d'étudier les différentes articulations de la main et les différents quadrants sans variation significative de la précision, contrairement aux études par échographie dans lesquelles les troisième et quatrième MCP sont peu accessibles sur les sites ulnaire et radial $[10,11]$.

Nous avons observé que la précision était plus élevée pour les érosions d'un diamètre axial supérieur à $1,9 \mathrm{~mm}$, comme illustré dans le tableau 1. Töpfer a décrit la même tendance, avec un rapport RMS CV/RMS SD de 3,02\%/0,92 mm pour les érosions de plus de $10 \mathrm{~mm}^{3}$, contre $6,11 \% / 0,32 \mathrm{~mm}^{3}$ pour les plus petites [21]. Cette taille $(\geq 1,9 \mathrm{~mm}$ ) avait été décrite comme la plus spécifique de la $P R$, des érosions plus petites pouvant être observées chez des sujets en bonne santé [21]. Une 
érosion spécifique de la PR devrait par conséquent être définie comme mesurant plus de $1,9 \mathrm{~mm}$ sur le scanner HRpQCT avant d'être utilisée comme cible thérapeutique. L'âge et la durée des symptômes étaient significativement corrélés à une augmentation de la RMS SD, ce qui a déjà été décrit pour la précision intraopérateur [22].

La qualité de la micro-architecture osseuse n'a pas eu d'incidence sur nos résultats de précision. De fait, on pouvait émettre l'hypothèse que la qualité de la microarchitecture était associée à une diminution de la précision. La délimitation des érosions devrait être plus difficile lorsque la différence de densité entre l'érosion et l'os trabéculaire adjacent est réduite. Une altération de la micro-architecture osseuse a été décrite au niveau du radius distal et ultra-distal dans la PR [16]. Par ailleurs, une dégradation du réseau trabéculaire et de l'épaisseur corticale a également été rapportée dans les articulations MCP, directement corrélée à l'activité de la maladie [17]. Cela étant, dans une population en bonne santé, le repositionnement n'a pas eu de répercussions sur la précision de la mesure de l'épaisseur des travées, de la séparation des travées et de l'épaisseur corticale, avec une RMC CV de 3,5\%, $1,3 \%$ et $2,7 \%$ respectivement [18].

Les résultats de reproductibilité à court terme étaient très similaires à ceux liés à la variabilité intra-opérateur, ce qui nous a incités à conclure que cette variabilité n'est pas due au repositionnement. D'une part, la technique manuelle introduit une subjectivité de l'opérateur et il a été montré que des divergences fortuites dans la délimitation des érosions étaient une source d'erreur majeure [27]. L'expérience du lecteur pourrait également contribuer à la variabilité des mesures. Dans cette étude, le premier auteur, formé par plusieurs membres du groupe SPECTRA, ne peut pas être considéré comme un lecteur inexpérimenté. Nos ICC étaient très similaires à ceux déjà publiés pour la précision intra-opérateur [6,21,22]. Nous avons également montré que l'entraînement ne semblait pas améliorer la précision car aucune différence n'a été constatée entre les résultats des premières et des dernières érosions évaluées (figure 4). De plus, la variabilité interopérateur était très proche des autres résultats, avec d'excellents ICC. Cette évaluation a été réalisée par le premier auteur et par le deuxième $(\mathrm{MH})$, lecteur expérimenté. L'effet de l'entraînement sur la reproductibilité interopérateur de la mesure manuelle des 
volumes d'érosion au niveau des MCP par IRM a déjà été étudié et jugé négligeable [37].

D'autre part, on ne peut pas affirmer avec certitude que la mesure des érosions a été réalisée exactement de la même manière après le repositionnement. La délimitation manuelle peut aussi prendre un certain temps, entre 1 minute pour la largeur et la profondeur et 5 minutes pour le volume. Cela a notamment été le cas chez les patients présentant de nombreuses érosions, en particulier lorsqu'elles étaient de grande taille ou situées dans deux quadrants différents. Pour les érosions non délimitées par de l'os sclérotique, l'évaluation de la profondeur ou du volume n'est pas facilement reproductible [21]. Cela étant, le recours au scanner HRpQCT ne serait pas utile dans le cas de grandes érosions détectables par la radiographie conventionnelle. Une autre difficulté technique a été liée à la présence d'artéfacts de mouvement, fréquents lorsque l'on utilise le $\mathrm{HRpQCT}$, en raison de la longue durée du scanner [18,21]. En outre, les patients atteints de PR présentant des érosions nombreuses et des subluxations articulaires avaient du mal à conserver la position.

La plus petite variation significative PPVS a été estimée à $0,22 \mathrm{~mm}$ pour la profondeur axiale, $0,33 \mathrm{~mm}$ pour le diamètre axial et $0,33 \mathrm{~mm}^{3}$ pour le volume. Elle a été calculée à partir des données avec repositionnement et n'était pas disponible dans les précédentes études. La plus petite variation détectable décrite par Finzel et al. a été estimée à $0,008 \mathrm{~mm}$ pour le diamètre axial et la profondeur axiale [7]. La même étude indiquait également une diminution moyenne de $0,11 \mathrm{~mm}$ du diamètre axial des érosions après un an de traitement par tocilizumab, ce qui ne dépasserait pas la PPVS de notre étude. II a été démontré que la réparation des lésions était associée à des appositions osseuses à la base des érosions [6]. Les études existantes n'ont pas mis en évidence de réparation significative dans les mesures de profondeur.

Les points forts de notre étude étaient le grand nombre d'érosions évaluées et la plage étendue de tailles mesurées. De plus, il s'agissait à notre connaissance de la première étude évaluant la reproductibilité à court terme avec repositionnement, ce qui marque une nouvelle étape vers l'introduction du scanner HRPQCT comme nouvelle technique d'imagerie de diagnostic et outil de surveillance dans la PR. Les limites étaient l'absence de technique de référence pour la mesure du volume et le petit nombre de patients, qui ne nous a pas permis de mettre en évidence des 
différences significatives concernant les sources de variation, notamment à propos des paramètres micro-architecturaux.

L'évaluation précise des paramètres de mesure des érosions est un critère essentiel pour décrire la réponse aux traitements dans la PR. Une nouvelle technique de segmentation tridimensionnelle a été mise au point récemment par Töpfer et al. [21]. Ils ont obtenu des résultats satisfaisants en termes de précision intra- et interopérateur avec une RMS CV de 5,66\% et de 7,76\% respectivement. II serait donc intéressant de mettre au point des techniques automatisées visant à améliorer la mesure des érosions par HRpQCT.

\section{Remerciements}

Les auteurs remercient tous les participants à l'étude. Ils expriment également leur reconnaissance à Cheryl Barnabe, Stéphanie Boutroy et au groupe SPECTRA pour leur aide scientifique, à Kathryn Stok et Stéphanie Rouanet pour leurs conseils en statistiques et à Évelyne Cordier pour son assistance technique.

\section{Déclaration d'intérêts}

Les auteurs n'ont aucun conflit d'intérêt à déclarer, ni aucun soutien financier ou autres avantages provenant de sources commerciales. 


\section{Références}

[1] Tobón GJ, Youinou P, Saraux A. The environment, geo-epidemiology, and autoimmune disease: Rheumatoid arthritis. J Autoimmun 2010;35:10-4.

[2] McGonagle D, Tan AL, Møller Døhn U, Ostergaard M, Benjamin M. Microanatomic studies to define predictive factors for the topography of periarticular erosion formation in inflammatory arthritis. Arthritis Rheum 2009;60:1042-51.

[3] van der Heijde D, Landewé R, van Vollenhoven R, Fatenejad S, Klareskog L. Level of radiographic damage and radiographic progression are determinants of physical function: a longitudinal analysis of the TEMPO trial. Ann Rheum Dis 2008;67:1267-70.

[4] Møller Døhn U, Boonen A, Hetland ML, et al. Erosive progression is minimal, but erosion healing rare, in patients with rheumatoid arthritis treated with adalimumab. A 1 year investigator-initiated follow-up study using high-resolution computed tomography as the primary outcome measure. Ann Rheum Dis 2009;68:1585-90.

[5] Walsh NC, Gravallese EM. Bone remodeling in rheumatic disease: a question of balance. Immunol Rev 2010;233:301-12.

[6] Finzel S, Rech J, Schmidt S, et al. Repair of bone erosions in rheumatoid arthritis treated with tumour necrosis factor inhibitors is based on bone apposition at the base of the erosion. Ann Rheum Dis 2011;70:1587-93.

[7] Finzel S, Rech J, Schmidt S, Engelke K, Englbrecht M, Schett G. Interleukin-6 receptor blockade induces limited repair of bone erosions in rheumatoid arthritis: a micro CT study. Ann Rheum Dis 2013;72:396-400.

[8] Van der Heijde DM. Radiographic imaging: the "gold standard" for assessment of disease progression in rheumatoid arthritis. Rheumatol Oxf Engl 2000;39 Suppl 1:9-16.

[9] Brower AC. Use of the radiograph to measure the course of rheumatoid arthritis. The gold standard versus fool's gold. Arthritis Rheum 1990;33:316-24.

[10] Finzel S, Ohrndorf S, Englbrecht M, et al. A detailed comparative study of highresolution ultrasound and micro-computed tomography for detection of arthritic bone erosions. Arthritis Rheum 2011;63:1231-6.

[11] Zayat AS, Ellegaard K, Conaghan PG, et al. The specificity of ultrasounddetected bone erosions for rheumatoid arthritis. Ann Rheum Dis 2015;74:897903.

[12] Farrant JM, Grainger AJ, O'Connor PJ. Advanced imaging in rheumatoid arthritis: part 2: erosions. Skeletal Radiol 2007;36:381-9.

[13] Døhn UM, Ejbjerg BJ, Court-Payen M, et al. Are bone erosions detected by magnetic resonance imaging and ultrasonography true erosions? A comparison with computed tomography in rheumatoid arthritis metacarpophalangeal joints. Arthritis Res Ther 2006;8:R110.

[14] Bird P, Lassere M, Shnier R, Edmonds J. Computerized measurement of magnetic resonance imaging erosion volumes in patients with rheumatoid arthritis: a comparison with existing magnetic resonance imaging scoring 
systems and standard clinical outcome measures. Arthritis Rheum 2003;48:61424.

[15] Emond PD, Inglis D, Choi A, Tricta J, Adachi JD, Gordon CL. Volume measurement of bone erosions in magnetic resonance images of patients with rheumatoid arthritis. Magn Reson Med Off J Soc Magn Reson Med Soc Magn Reson Med 2012;67:814-23.

[16] Boutroy S, Bouxsein ML, Munoz F, Delmas PD. In vivo assessment of trabecular bone microarchitecture by high-resolution peripheral quantitative computed tomography. J Clin Endocrinol Metab 2005;90:6508-15.

[17] Fouque-Aubert A, Boutroy S, Marotte $\mathrm{H}$, et al. Assessment of hand bone loss in rheumatoid arthritis by high-resolution peripheral quantitative CT. Ann Rheum Dis 2010;69:1671-6.

[18] Feehan L, Buie H, Li L, McKay H. A customized protocol to assess bone quality in the metacarpal head, metacarpal shaft and distal radius: a high resolution peripheral quantitative computed tomography precision study. BMC Musculoskelet Disord 2013;14:367.

[19] Stach CM, Bäuerle M, Englbrecht M, et al. Periarticular bone structure in rheumatoid arthritis patients and healthy individuals assessed by high-resolution computed tomography. Arthritis Rheum 2010;62:330-9.

[20] Barnabe C, Szabo E, Martin L, Boyd SK, Barr SG. Quantification of small joint space width, periarticular bone microstructure and erosions using high-resolution peripheral quantitative computed tomography in rheumatoid arthritis. Clin Exp Rheumatol 2013;31:243-50.

[21] Töpfer D, Finzel S, Museyko O, Schett G, Engelke K. Segmentation and quantification of bone erosions in high-resolution peripheral quantitative computed tomography datasets of the metacarpophalangeal joints of patients with rheumatoid arthritis. Rheumatol Oxf Engl 2014;53:65-71.

[22] Srikhum W, Virayavanich W, Burghardt AJ, et al. Quantitative and semiquantitative bone erosion assessment on high-resolution peripheral quantitative computed tomography in rheumatoid arthritis. J Rheumatol 2013;40:408-16.

[23] Buckland-Wright JC. Microfocal radiographic examination of erosions in the wrist and hand of patients with rheumatoid arthritis. Ann Rheum Dis 1984;43:160-71.

[24] C. Barnabe SK. FRI0249 Case Definition for Erosions Imaged with High Resolution Peripheral Quantitative Computed Tomography (HR-PQCT): an International Spectra Reliability Exercise-1 (RELEX-1). Ann Rheum Dis 2014;73:473-4.

[25] Finzel S, Englbrecht M, Engelke K, Stach C, Schett G. A comparative study of periarticular bone lesions in rheumatoid arthritis and psoriatic arthritis. Ann Rheum Dis 2011;70:122-7.

[26] Boutroy S, Chapurlat R, Vanden-Bossche A, Locrelle H, Thomas T, Marotte H. Erosion or Vascular Channel? Arthritis Rheumatol 2015;67:2956-2956.

[27] Døhn UM, Ejbjerg BJ, Hasselquist M, et al. Rheumatoid arthritis bone erosion volumes on CT and MRI: reliability and correlations with erosion scores on $\mathrm{CT}$, MRI and radiography. Ann Rheum Dis 2007;66:1388-92.

[28] Giraudeau B, Gomez MA, Defontaine M. Assessing the reproducibility of quantitative ultrasound parameters with standardized coefficient of variation or intraclass correlation coefficient: a unique approach. Osteoporos Int J Establ 
Result Coop Eur Found Osteoporos Natl Osteoporos Found USA 2003;14:6145.

[29] Glüer CC, Blake G, Lu Y, Blunt BA, Jergas M, Genant HK. Accurate assessment of precision errors: how to measure the reproducibility of bone densitometry techniques. Osteoporos Int $\mathrm{J}$ Establ Result Coop Eur Found Osteoporos Natl Osteoporos Found USA 1995;5:262-70.

[30] Grampp S, Lang P, Jergas M, et al. Assessment of the skeletal status by peripheral quantitative computed tomography of the forearm: short-term precision in vivo and comparison to dual X-ray absorptiometry. J Bone Miner Res Off J Am Soc Bone Miner Res 1995;10:1566-76.

[31] Martin Bland J, Altman D. Statistical methods for assessing agreement between two methods of clinical measurement. The Lancet 1986;327:307-10.

[32] Giavarina D. Understanding Bland Altman analysis. Biochem Medica 2015;25:141-51.

[33] Leslie WD, Moayyeri A, Manitoba Bone Density Program. Minimum sample size requirements for bone density precision assessment produce inconsistency in clinical monitoring. Osteoporos Int J Establ Result Coop Eur Found Osteoporos Natl Osteoporos Found USA 2006;17:1673-80.

[34] Genant HK, Jergas M, Palermo L, et al. Comparison of semiquantitative visual and quantitative morphometric assessment of prevalent and incident vertebral fractures in osteoporosis The Study of Osteoporotic Fractures Research Group. J Bone Miner Res Off J Am Soc Bone Miner Res 1996;11:984-96.

[35] Barnabe C, Feehan L, SPECTRA (Study GrouP for XTrEme-CT in RA). Highresolution peripheral quantitative computed tomography imaging protocol for metacarpophalangeal joints in inflammatory arthritis: the SPECTRA collaboration. J Rheumatol 2012;39:1494-5.

[36] Tan AL, Tanner SF, Conaghan PG, et al. Role of metacarpophalangeal joint anatomic factors in the distribution of synovitis and bone erosion in early rheumatoid arthritis. Arthritis Rheum 2003;48:1214-22.

[37] Bird P, Joshua F, Lassere M, Shnier R, Edmonds J. Training and calibration improve inter-reader reliability of joint damage assessment using magnetic resonance image scoring and computerized erosion volume measurement. J Rheumatol 2005;32:1452-8. 


\section{Légende des figures}

Figure 1. Mesure d'une érosion d'une troisième tête métacarpienne A : largeur axiale et profondeur axiale ; B : largeur perpendiculaire et profondeur perpendiculaire (plan frontal) ; $\mathrm{C}$ : volume

Figure 2. Répartition des érosions dans les têtes métacarpiennes et les bases phalangiennes selon les quadrants et les articulations définis

TM : tête métacarpienne ; BP : base phalangienne En abscisse, l'emplacement par quadrant et par articulation. En ordonnée, le rapport entre nombre d'érosions par quadrant et nombre total d'érosions (406).

Figure 3. Graphiques de Bland et Altman représentant la précision avec repositionnement pour la mesure de 406 érosions

Chaque graphique présente en abscisse la moyenne des deux mesures après repositionnement et en ordonnée, la différence entre les deux mesures après le repositionnement. La ligne centrale représente la moyenne de la différence des deux mesures et les lignes périphériques, les limites d'agrément équivalentes à 1,96 ET de la moyenne de la différence des deux mesures.

\section{Figure 4. Racine carrée de l'écart-type par patient}

Les patients et la racine carrée de l'écart-type (RMS SD) par valeur de patient sont représentés respectivement en abscisse et en ordonnée. wa : largeur axiale ; da : profondeur axiale ; wp : largeur perpendiculaire ; dp : profondeur perpendiculaire ; vol : volume 

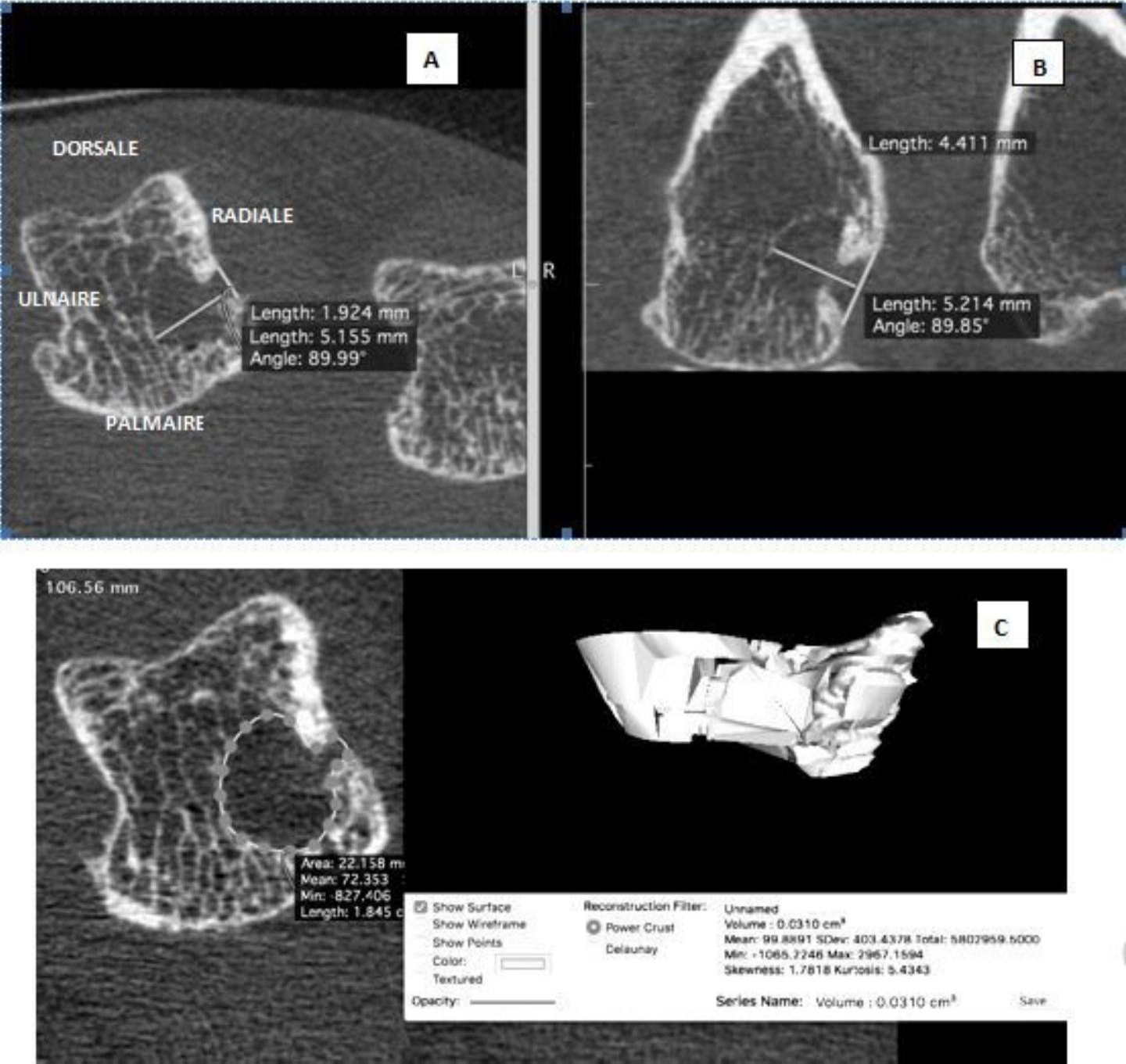


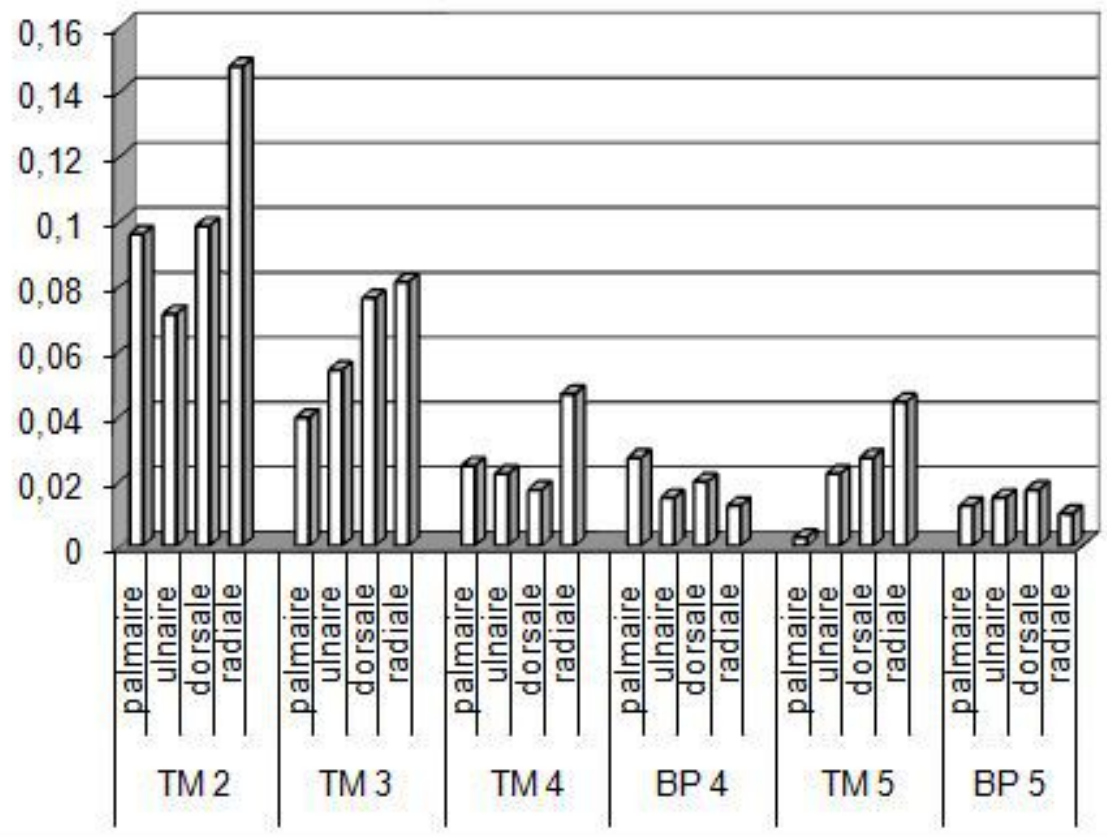



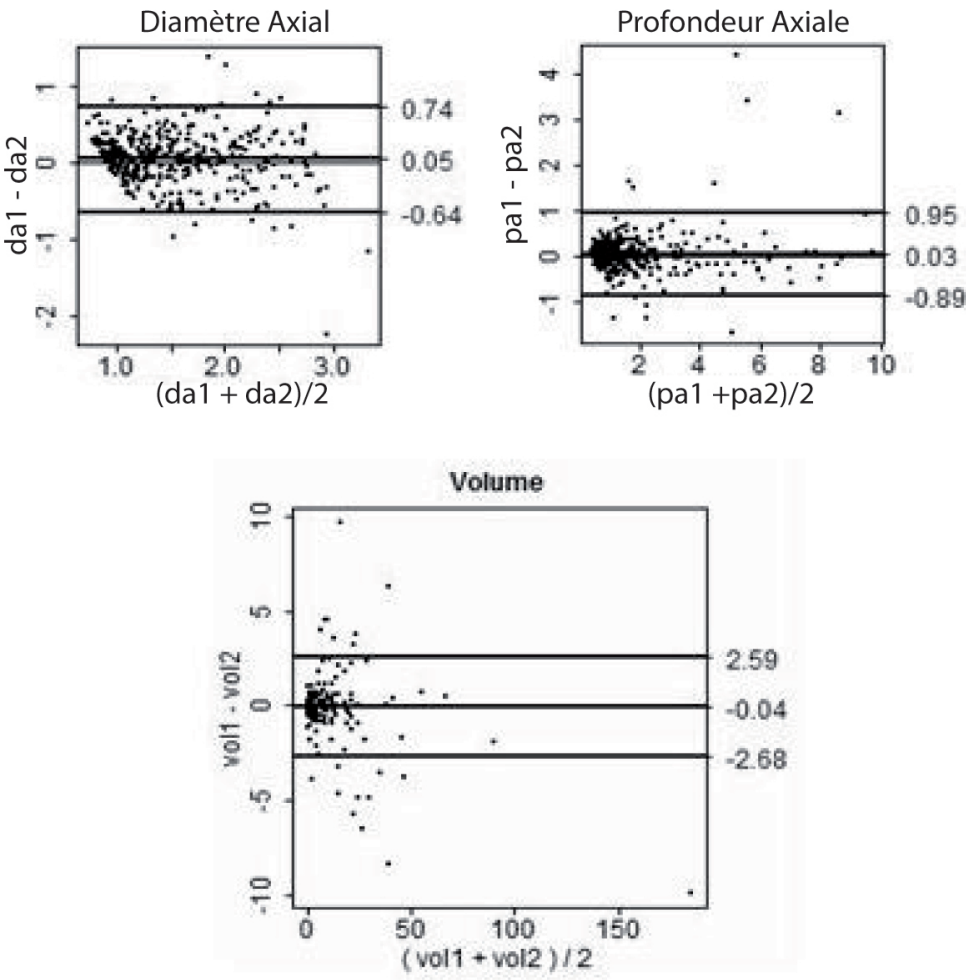
Tableau 1. Reproductibilité intra observateur à court terme après repositionnement

\begin{tabular}{|c|c|c|c|c|c|c|}
\hline & Précision & $\begin{array}{l}\text { Diamètre } \\
\text { axial }\end{array}$ & $\begin{array}{l}\text { Profondeur } \\
\text { axiale }\end{array}$ & $\begin{array}{l}\text { Diamètre } \\
\text { perpendiculaire }\end{array}$ & $\begin{array}{l}\text { Profondeur } \\
\text { perpendiculaire }\end{array}$ & $\begin{array}{l}\text { Volume } \\
\left(\mathrm{mm}^{3}\right)\end{array}$ \\
\hline \multirow{4}{*}{$\begin{array}{c}\text { Toutes } \\
\text { les } \\
\text { érosions } \\
n=406\end{array}$} & ICC & 0,80 & 0,96 & 0,86 & 0,91 & 0,99 \\
\hline & $\begin{array}{c}\text { RMS CV } \\
\%\end{array}$ & 16,0 & 17,5 & 20,0 & 23,5 & 19,7 \\
\hline & RMS SD & & & & & \\
\hline & (mm) & 0,25 & 0,31 & 0,30 & 0,47 & 1,66 \\
\hline $\begin{array}{l}\text { Érosions } \\
<1,9 \mathrm{~mm}\end{array}$ & $\begin{array}{c}\text { RMS CV } \\
\%\end{array}$ & 16,6 & 18,6 & 20,9 & 25 & 21,2 \\
\hline $\mathrm{n}=\mathbf{3 0 5}$ & $\begin{array}{c}\text { RMS SD } \\
(\mathrm{mm})\end{array}$ & 0,22 & 0,29 & 0,27 & 0,49 & 0,67 \\
\hline $\begin{array}{l}\text { Érosions } \\
>1,9 \mathrm{~mm}\end{array}$ & $\begin{array}{c}\text { RMS CV, } \\
\%\end{array}$ & 16,6 & 13,5 & 16,9 & 17,7 & 14 \\
\hline$n=101$ & $\begin{array}{c}\text { RMS SD } \\
(\mathbf{m m})\end{array}$ & 0,34 & 0,41 & 0,39 & 0,58 & 1,45 \\
\hline
\end{tabular}

RMS CV : racine carrée du coefficient de variation ; RMS SD : racine carrée de l'écart-type ; ICC : coefficient de corrélation intraclasse 
Tableau 2. Corrélations entre RMS SD et données cliniques et de micro-architecture osseuse.

\begin{tabular}{|c|c|c|c|c|c|c|c|c|c|c|}
\hline & Âge & $\begin{array}{l}\text { Durée des } \\
\text { symptômes }\end{array}$ & $\begin{array}{c}\text { DAS } \\
28\end{array}$ & $\begin{array}{c}\text { Nombre } \\
\text { d'érosions }\end{array}$ & $\begin{array}{c}\text { Diamètre } \\
\text { axial }\end{array}$ & $\mathrm{BV} / \mathrm{TV}$ & Tb.N & Tb.Th & $\mathrm{Tb} . \mathrm{Sp}$ & Ct.Th \\
\hline $\begin{array}{l}\text { RMS SD } \\
\text { Diamètre } \\
\text { axial }\end{array}$ & $0,44^{\star}$ & 0,22 & 0,07 & 0,31 & $\mathbf{0 , 4 8} 8^{\star *}$ & $-0,11$ & $-0,15$ & 0,07 & $-0,01$ & $-0,22$ \\
\hline $\begin{array}{l}\text { RMS SD } \\
\text { profondeur } \\
\text { axiale }\end{array}$ & $0,40^{*}$ & 0,17 & 0,06 & $0,55^{\star \star}$ & $0,38^{*}$ & $-0,35$ & $-0,32$ & 0,35 & 0,32 & $-0,33$ \\
\hline $\begin{array}{l}\text { RMS SD } \\
\text { Diamètre } \\
\text { perp. }\end{array}$ & 0,24 & $0,39^{*}$ & $0, \overline{05}$ & $0,41^{*}$ & 0,28 & $-0,28$ & $-0,18$ & 0,11 & 0,29 & $-0,36$ \\
\hline $\begin{array}{l}\text { RMS SD } \\
\text { profondeur } \\
\text { perp. }\end{array}$ & 0,29 & 0,19 & 0,25 & $0,60^{\star \star \star}$ & $0,52^{\star \star}$ & $-0,18$ & $-0,21$ & 0,27 & 0,26 & $-0,28$ \\
\hline $\begin{array}{l}\text { RMS SD } \\
\text { volume }\end{array}$ & 0,14 & $0,46^{\star \star}$ & 0,20 & 0,35 & $0,77^{\star \star \star}$ & $-0,29$ & $-0,20$ & 0,09 & 0,31 & $-0,17$ \\
\hline
\end{tabular}

RMS SD : racine carrée de l'écart-type ; perp. : perpendiculaire ; BV/TV : volume osseux/volume tissulaire ; Tb.N : nombre de travées ; Tb.Th : épaisseur des travées ; Tb.Sp : séparation des travées; Ct.Th : épaisseur corticale ; $p=0,05 ;{ }^{* *} p \leq 0,05 ;{ }^{* * *} p \leq 0,005$. 